\title{
Crise de l'état et nouvelles autorités : les juntes lors de la guerre d'indépendance
}

\section{Antonio Moliner Prada}

\section{(2) OpenEdition \\ 1 Journals}

\section{Édition électronique}

URL : https://journals.openedition.org/ahrf/1691

DOI : 10.4000/ahrf.1691

ISSN : 1952-403X

Éditeur :

Armand Colin, Société des études robespierristes

\section{Édition imprimée}

Date de publication : 1 juin 2004

Pagination : 107-128

ISSN : 0003-4436

Référence électronique

Antonio Moliner Prada, «Crise de l'état et nouvelles autorités : les juntes lors de la guerre

d'indépendance ", Annales historiques de la Révolution française [En ligne], 336 | avril-juin 2004, mis en ligne le 15 juillet 2007, consulté le 24 avril 2022. URL : http://journals.openedition.org/ahrf/1691 ; DOI : https://doi.org/10.4000/ahrf.1691

Ce document a été généré automatiquement le 24 avril 2022.

Tous droits réservés 


\title{
Crise de l'état et nouvelles autorités : les juntes lors de la guerre d'indépendance ${ }^{1}$
}

\author{
Antonio Moliner Prada
}

1 Après la signature du Traité de Fontainebleau (27 octobre 1807), les troupes françaises traversèrent la frontière et occupèrent progressivement le territoire espagnol pendant les premiers mois de 1808. Les institutions du pays, la Junte Suprême de Gouvernement, nouvellement créée, et le Conseil de Castille furent d'une inefficacité absolue, se limitant à prôner le pacifisme face à l'invasion. Les capitaines généraux et les Audiences essayèrent par tous les moyens d'apaiser les esprits et de maintenir le statu quo dans toutes les provinces.

2 La situation d'anarchie croissante incita les patriotes à chercher une issue nouvelle dans le but de résoudre la crise politique, créant les Juntes d'autorités dans les villes et dans les provinces. Pour comprendre en profondeur le processus de leur formation il nous faut tenir compte de l'état d'insécurité existant dans l'ensemble du pays. Il suffirait de voir les mutineries et les révoltes qui se produisirent, de mai à juin, presque toujours de manière spontanée, dont l'esprit anti-Godoy et xénophobe était patent ${ }^{2}$.

On ne peut dissocier la formation des Juntes et le soulèvement populaire, bien que les nouvelles institutions créées aient été formées la plupart du temps par les membres des élites locales et provinciales et non pas directement par le peuple. La société continue de se concevoir suivant l'imaginaire de l'Ancien Régime, c'est-à-dire celui des différents états. C'est la raison pour laquelle ceux-ci sont tous représentés dans les Juntes et que l'on fait appel aux institutions traditionnelles comme la Junte Générale de la Principauté des Asturies, les Cortès d'Aragon, etc. ${ }^{3}$.

4 Les Juntes se sont formées en marge du pouvoir constitué, ou parfois en lui faisant face. Pouvoir qui d'ailleurs n'existe plus puisque le roi en est absent. Une rupture se produisit dans ce sens avec les autorités établies. Suivant la doctrine préconisant le pacte, les Juntes étaient à présent les dépositaires de la souveraineté, aspect profondément révolutionnaire. Dans un sens, comme le signale Flórez Estrada dans le 
cas de la Junte des Asturies, la souveraineté est retournée à nouveau au peuple où elle réside en permanence " surtout lorsque la personne qui l'a cédé n'existe plus $»^{4}$.

Les dix-huit Juntes Suprêmes Provinciales qui se sont constituées apparaissent comme des pouvoirs révolutionnaires en se proclamant souveraines grâce au pouvoir qu'elles avaient reçu du peuple et, agissant au nom de Ferdinand, ne reconnaissent pas les abdications de Bayonne, fruit de la violence. Elles se chargent de titres et d'honneurs, cherchant leur légitimité rituelle et agissent de ce fait avec une indépendance totale : elles organisent la résistance et l'armée, nomment des généraux et d'autres fonctionnaires, lèvent des impôts, administrent les rentes et établissent des relations avec d'autres pays et entre les Juntes elles-mêmes. Leur objectif principal est d'établir un plan de défense dans chaque territoire pour conserver l'indépendance de la nation. C'est pourquoi, à de nombreuses reprises, il se produisit des heurts avec les cadres militaires. En effet, ils se voyaient souvent obligés d'entreprendre des actions d'une efficacité militaire pour le moins douteuse, contraints par la suprématie politique du pouvoir civil.

6 Les circonstances particulières à chaque territoire ou à chaque ville étaient différentes, c'est pourquoi nous trouvons une typologie de Juntes aussi diverse. Il existait dans certaines villes des petits groupes de patriotes qui se réunissaient bien avant le soulèvement, comme à La Corogne et dans les Asturies. On peut même penser que dans d'autres villes il ait pu y avoir des éléments actifs du parti fernandin ou aristocratique disposés à contrôler la nouvelle situation. Nous n'avons toutefois pas assez de preuves pour pouvoir parler d'une planification du soulèvement anti-français, pas plus que nous ne savons si la formation des Juntes obéissait à un modèle fixé d'avance ${ }^{5}$. Dans certaines Juntes c'étaient les militaires qui prédominaient alors que dans d'autres c'étaient les anciennes autorités, parmi lesquelles on trouvait les membres de l'aristocratie nobiliaire unis par des liens de famille et de clientélisme ${ }^{6}$. Les militaires, simples gradés ou officiers supérieurs qui font partie des Juntes, manquent d'enthousiasme et de pouvoir, la comparution volontaire des généraux étant tout à fait exceptionnelle. La composition des Juntes est fort hétérogène : autorités provinciales ou régisseurs locaux, membres de l'armée, de l'administration et des corporations, ecclésiastiques, chanoines, évêques, nobles, bourgeois et opportunistes de tout ordre luttant pour le pouvoir. A. Von Schepeler signale à juste titre que de nombreux anciens fonctionnaires, ecclésiastiques et nobles, hostiles à toute sorte de révolution, en firent partie et les utilisèrent pour contrôler l'explosion populaire ${ }^{7}$. Parallèlement, les particularismes locaux et la rivalité entre les différentes localités s'exacerbent. Les Juntes dans leur ensemble se sentent toutefois solidaires et manifestent un fort sentiment national que l'on observe davantage dans la littérature politique de l'époque que dans leurs proclamations ${ }^{8}$.

7 S'agissant d'un soulèvement national et patriotique on peut trouver parmi les membres des Juntes des personnes aux caractères différents et d'idéologie antagoniste, notamment des absolutistes, mais parfois aussi des libéraux. Ne soyons pas tentés de minimiser l'importance de cette minorité libérale qui devait jouer ensuite un rôle majeur. Romero Alpuente ou Antillón, membre de la Junte de Teruel, Calvo de Rozas qui représenta l'Aragon à la Junte Centrale, Flórez Estrada, dont nous avons déjà parlé, membre de la Junte des Asturies, le vicomte de Quintanilla de la Junte de León, l'évêque Nadal, de la Junte de Majorque ou les Bertrán de Lis, de la Junte de Valence. 
8 Les obligations militaires et de centralisation obligèrent les Juntes à créer, le 25 septembre 1808, une Junte Centrale évitant ainsi, ce que l'on appelait à l'époque "l'hydre du fédéralisme». Le principe d'unité et de hiérarchie triompha sur les idées des Juntes de Galice, de Castille et León unies et celles de Grenade et de Valence qui voulaient faire de la Junte Centrale un organisme dépendant des provinces, limitant la permanence et l'action de ses députés. Bien que ses fonctions fussent peu définies dans son Règlement pour le gouvernement intérieur, elle exerça le pouvoir consciente d'être la plus haute autorité et que ses membres étaient les représentants, non pas de leurs provinces respectives, mais de la nation toute entière. C'est pourquoi elle obligea les autorités constituées à lui prêter obéissance et exigea au Conseil de Castille un décret ordonnant de lui concéder le traitement de majesté. Elle reconnut la dette nationale, imposa un impôt de guerre extraordinaire et signa un traité d'alliance avec le Royaume-Uni en janvier 1809.

9 La Junte Centrale limita les pouvoirs des Juntes provinciales. Bien qu'elle reconnaissaît les services prêtés dans le but de sauvegarder l'unité nationale, elle leur interdit la possibilité de concéder des grades militaires et des fonctions, aussi bien civiles qu'ecclésiastiques. Elle créa la figure des commissaires de la Junte suprême Gouvernementale du Royaume dans les provinces (R.O. du 16 octobre 1808) et leur attribua de larges compétences: activer les équipements de guerre, rapprocher le pouvoir central des Juntes et du peuple, concilier les divergences pouvant exister entre les Juntes et les autorités militaires. Le Règlement sur les Juntes provinciales (1er janvier 1809) diminua leur influence et réduisit leurs compétences et attributions, en introduisant un plan uniforme dans le gouvernement et l'administration des provinces. Les Juntes perdaient ainsi leur ascendant initial et leurs attributions; elles en vinrent à jouer un rôle de simples organismes intermédiaires entre le peuple et les autorités. Le signe et la preuve évidente de ce changement en sont leur nouvelle dénomination (Juntes supérieures provinciales d'observation et de défense), leur hiérarchie (Suprême $\mathrm{du}$ Royaume, Juntes provinciales, Juntes de chef-lieu), ainsi que la tendance à réduire leurs membres (9 dans les provinciales et 5 dans celles de chefs-lieux), s'abstenant de toute manifestation de juridiction et d'autorité n'étant pas encadrée dans ce Règlement. Avec celui-ci triomphe définitivement le critère centraliste, ce qui est logique en temps de guerre, les Juntes provinciales n'étant plus que de simples instruments d'exécution des ordres émanant de la Centrale.

10 Il y a eu différentes interprétations des Juntes (ultraconservatrice, marxiste ou fédéraliste-régionaliste) ${ }^{9}$. Un risque existe d'utiliser un schéma explicatif simpliste et linéaire. On ne peut pas parler de révolution populaire, car le peuple est absent des Juntes. Mais on ne peut pas analyser ces dernières sans le soulèvement populaire qui précéda leur formation. Leurs résolutions sont en partie contradictoires et ambiguës, elles n'ont jamais prétendu changer l'ordre social en place, mais, du fait de circonstances particulières, les Juntes s'étant dotées de pouvoirs, elles ouvrirent le processus politique qui aboutit à l'œuvre des Cortès de Cadix et à la proclamation de la Constitution de 1812.

11 Le but de cet article est de réviser, de manière critique, les recherches menées ces dernières années au sujet des Juntes pour établir ainsi un état de la question. Il faut tout de suite signaler l'énorme difficulté qui existe lorsqu'il faut créer un modèle interprétatif exprimant les traits particuliers des différentes Juntes et, notamment, lorsque le nombre de leurs membres changeait sans cesse et leurs prises de décisions 
évoluaient suivant les circonstances et les territoires. Prétendre fixer une image statique et totalement définie des Juntes oblige à se contraindre à un schéma fixé au préalable qui s'avère irréel. Faire une étude sans consulter leurs Actes et documents, dispersés dans les différentes archives à travers toute l'Espagne, ne peut que mener à élaborer de toutes pièces une interprétation falsifiée.

La Junte d'Asturies

Cette Junte fut la première à s'établir et c'est là la raison pour laquelle elle eut tant d'importance et servit de modèle à d'autres provinces. Après les divers incidents qui eurent lieu à Gijón et à Oviedo en avril, un groupe de patriotes auquel s'unirent des étudiants des universités et des ouvriers qui s'étaient emparés des fusils d'une usine d'armes réussirent à ce que la traditionnelle Junte Générale se réunisse le 9 mai dans la capitale de la Principauté des Asturies. Il fallut attendre le 25 mai pour que la nouvelle Junte se forme, et cela sous la pression de ce groupe de patriotes, avec l'appui des paysans en armes. La Junte, formée en marge de l'ancienne institution traditionnelle de la Principauté et de l'Audience, assuma à nouveau la souveraineté au nom de Ferdinand VII. Parmi ses membres apparaissent six membres de la noblesse face à une minorité libérale dirigée par Alvaro Flórez Estrada. Bientôt se fit jour l'opposition des autorités ecclésiastiques et de l'Audience chacune essayant de manœuvrer dans leur intérêt, modifiant pour cela sa composition. L'action d'Alvaro Flórez Estrada du 13 juin, qui réussit à ce que la Junte approuve un document révolutionnaire convoquant les Cortès, fut tout à fait surprenante.

13 La Junte des Asturies fut dissoute par le marquis de la Romana en mai 1809 et, à la demande de la Centrale, une nouvelle fut formée le 4 mars 1810, composée par 19 membres qui, plus tard, se réduisirent à 9 , suivant les instructions du conseil de Régence ${ }^{10}$.

Les Juntes d'Andalousie

14 Séville vécut trois jours d'agitation lorsque se propagea la nouvelle informant que les autorités acceptaient l'invasion française et que l'armée de Dupont avançait vers l'Andalousie. Les artisans et les ouvriers journaliers venant des quartiers extérieurs de la ville s'approprièrent des armes de la Real Maestranza de Artillería ${ }^{11}$. La Junte de Séville apparaît, suivant certains de ses membres - et les versions officielles de l'époque le confirment -, comme l'organisme qui sauva la ville de la révolution populaire. Elle fut formée le 27 mai 1808, sous la présidence de M. Francisco Saavedra qui prit le nom de Suprême d'Espagne et des Indes. Notons que parmi ses objectifs prioritaires il y avait l'aide à l'extension du soulèvement anti-napoléonien dans le Sud-Est espagnol.

Les 21 membres élus pour la Junte représentaient l'ancienne administration: cinq ecclésiastiques, deux nobles, deux militaires, deux commerçants, trois membres de l'Audience territoriale, quatre de la corporation municipale et, pour le reste, des personnalités de la ville. Si le caractère de cette Junte était, de toute évidence, conservateur, il ne faut pas perdre de vue le Manifeste du 3 août 1808 qui demandait la solidarité de toutes les autres Juntes Suprêmes dans le but de constituer un gouvernement civil fort, face à une dictature militaire contraire à la tradition historique de l'Espagne. Cette Junte fut une Junte arrogante qui s'attribua de nombreuses prérogatives et qui eut des problèmes de relations avec celles d'Andalousie, Jaén et Grenade, pour n'en citer que quelques-unes. La Junte de Séville, reconstituée le 24 janvier 1810, après la création du Conseil de Régence fut transférée 
sept jours plus tard dans la ville d'Ayamonte, près de Huelva et s'érigea comme commandement de l'armée espagnole du Sud-Ouest.

À Huelva, où le Conseil municipal voulut justifier la création d'une Junte, la Junte de Séville en rejeta la proposition arguant qu'il manquait «les deux mille habitants, et plus » nécessaires. Ayamonte se mit à la disposition de Séville et seulement un petit nombre de personnes s'adjoignirent à sa municipalité pour le gouvernement de la ville. C'est pourquoi aucun changement politique majeur n'eut lieu: tout était resté aux mains des mêmes. Ceux qui furent appelés les «acompañados» (trois individus) et six députés nommés, furent élus parmi les fils de l'élite locale, représentants de la noblesse, de la marine et du clergé ${ }^{12}$.

17 À Jaén, on forma le 30 mai une Junte de Sécurité Publique pour calmer l'état d'anxiété existant dans le peuple. M. Antonio Ponce de Léon, duc de Montemar et comte de Garcíez, la présida. Parmi les autres membres, on trouvait l'intendant de la province, le commandant général, deux représentants de l'évêque, deux du Chapitre ecclésiastique, deux de la municipalité et le syndic procureur général et le syndic mandataire en représentation du peuple ${ }^{13}$.

$\mathrm{Au}$ début du mois de juillet 1808, la nouvelle Junte prit le nom de Junte Suprême Gouvernementale du Royaume de Jaén, elle comprenait trente-six membres (huit ecclésiastiques, huit nobles, quatre militaires, quatre propriétaires terriens et le reste des membres de l'administration municipale ou provinciale). Si les relations entre la Junte de Jaén et celles de Cordoue et de Grenade furent purement protocolaires et peu fréquentes, il n'en fut pas de même avec celle de Séville. Celle-ci essaya d'imposer son mandataire, le capitaine de navire M. Manuel de Torres Valdivia, pour qu'il en occupe la présidence dans le but de chercher l'unité et une coordination plus poussée. La Junte de Jaén refusa avec fermeté cette motion et proposa finalement à celle de Séville que ledit mandataire fut nommé Commandant Général des Armes de la Province de Jaén.

La Junte de Grenade se constitua le 30 mai par la pression populaire et cela en dépit du manque d'enthousiasme initial de son capitaine général, $M$. Ventura Escalante. On est surpris par le grand nombre de membres qui faisaient partie de la Junte, exactement quarante et un : quatre appartenant à l'état militaire, le capitaine général compris, dixhuit au clergé avec à sa tête l'évêque, sept de l'Audience, deux à la noblesse, trois aux corporations et trois à l'administration municipale, outre un certain nombre de notables ${ }^{14}$.

20 La Junte de Grenade ne reconnut la suprématie imposée par la Junte de Séville que le 11 juin 1808, lorsqu'elles signèrent une convention reconnaissant que Grenade acceptait que son armée fut commandée par le général de la Junte sévillane, le général Castaños. Les mésententes entre celles-ci ressurgirent cependant après la Bataille de Bailen, lorsque les honneurs échurent aux généraux Castaños et Peña, et non pas à Reding, qui avait été nommé par la Junte de Grenade général en chef des troupes de son Royaume.

21 Vers la fin mai, Malaga constitua une Junte Suprême de Gouvernement où étaient représentés les différents ordres de la ville : trois ecclésiastiques, parmi eux l'évêque José Vicente Lamadrid, deux autorités royales, trois membres de la municipalité, un militaire, deux représentants de l'administration et deux autres personnalités. Présidée par Teodoro Reding, elle s'est immédiatement opposée à tout type de violence ou d'outrages, mais elle fut incapable d'éviter les meurtres de Juan Croharé et du viceconsul français d'Agaud. La relation entre la Junte de Grenade et celle de Malaga fut loin d'être cordiale. Elle ne vit jamais d'un bon œil le désir de supériorité dont Grenade 
faisait preuve. Cette question fut portée devant la Junte Centrale, mais la résolution ne lui fut pas favorable.

Les membres de la Junte de Malaga et le général Cuesta furent démis de leurs fonctions en 1810 par un groupe de mutins qui n'étaient pas disposés à se rendre aux Français comme le voulaient les autorités dirigées par le chanoine Jiménez, le prêtre Fernando de Berocal et un ex-capucin. Le 5 février après-midi, après une lutte acharnée dans les rues, la ville tomba aux mains des impériaux qui s'adonnèrent à des actes de vandalisme et de pillage, ce qu'ils avaient déjà fait dans de nombreuses autres villes ${ }^{15}$. Cordoba créa une Junte de tranquillité le 28 mai, à la demande de l'envoyé de la Junte sévillane Ramón Gavilanes, formée par sept membres (deux représentants de l'autorité royale, trois du corps municipal, un militaire et un ecclésiastique). Cadix se souleva à la demande du comte de Tebas et, après avoir assassiné le général Francisco Solano, une Junte se constitua le 30 mai et reconnut celle de Séville comme étant Suprême. Parmi les dix-huit membres de la Junte de Cadix, il y avait deux représentants de l'autorité royale, sept autorités de la municipalité, quatre militaires, trois ecclésiastiques et deux de l'administration provinciale. Finalement, la Junte d'Almeria fut constituée avec huit individus : deux représentants de l'autorité royale, un de la municipalité, un militaire, trois ecclésiastiques et un représentant des corporations.

La création par la Junte Centrale, en décembre 1808, de la Junte appelée Junte pour la défense des Royaumes d'Andalousie et la Manche pour défendre les passages de Sierra Morena, ne manque pas de nous surprendre. Cette Junte s'est constituée tout d'abord à Cordoba avec la participation de l'Estrémadure au lieu de la Manche. Deux représentants des Juntes de Séville, Cordoba, Grenade et Jaén y participèrent, deux autres de celle d'Estrémadure et deux de la Centrale. Peu après sa création elle s'établit dans la Carolina jusqu'à ce que les Français entrent dans cette ville.

Les Juntes d'Estrémadure

À Badajoz, le 30 mai, jour de l'anniversaire du roi Ferdinand, le peuple se mutina et assassina le capitaine général comte Torre del Fresno, cousin de Godoy. Son substitut, le général Galluzo, constitua, tout de suite après, une Junte intérimaire. La première session de la Junte s'est tenue le 31 mai et le capitaine Ramón Gavilanes, envoyé par la Junte de Séville pour organiser le mouvement, joua un rôle prédominant. À Caceres, on fit circuler un pasquin contre l'Audience le 31 mai et les autorités décidèrent lors d'une réunion de former une Junte de Gouvernement dont le seul objectif était de contrôler l'ordre public et d'éviter des incidents sanglants comme ceux ayant eu lieu à Badajoz. Ce fut la pression populaire qui força la Junte de Caceres à ordonner le recrutement général des hommes entre 16 et 40 ans. À Plasencia se produisirent aussi plusieurs assassinats d'individus accusés de trahison et l'on constitua une junte d'Armement et de Défense, présidée par l'évêque du diocèse.

Au début du mois de juin, on constitua la Junte d'Estrémadure à partir de la première Junte de Badajoz, qui incorpora des représentants de toute la province, et celle de Caceres dont le nombre de membres changea progressivement tout au long de ses quatre ans d'existence, allant jusqu'à quarante-deux. Au début, elle fut présidée par l'évêque de Badajoz et, plus tard, par des militaires. On peut s'étonner des difficiles relations existant entre la Junte d'Estrémadure et le marquis de la Romana ainsi que du manque de représentation de certains partis de la Junte. Celle-ci se vit obligée d'imposer une amende de mille ducats au comte de Canilleros, représentant du parti de 
Alcantara, pour avoir rapporté des allégations sans fondement dans le but d'échapper à ses obligations patriotiques ${ }^{16}$.

Les Juntes d'Aragon

À Saragosse, des agitations populaires eurent lieu de mars à mai. En avril, il y eut des émeutes d'étudiants et le 24 mai ce fut le tour des paysans. Toutes les sources décrivent le déroulement de ces faits de manière semblable: l'attente du courrier, la cocarde rouge comme signal, la mise aux arrêts du capitaine général Guillelmi, l'assaut au château de l'Aljafería et la distribution d'armes. Les autorités royales ayant été destituées à la suite de l'insurrection, le jeune brigadier José Palafox fut nommé chef de l'armée par le peuple de Saragosse. Le 27 mai, il réunit une Junte qui accorda les premiers recrutements et la création de milices. Pour légitimer le soulèvement, il convoqua les Cortès le 9 juin. Trente-trois députés y assistèrent, le confirmèrent dans son commandement et élirent une Junte de gouvernement de six individus (trois nobles, un ecclésiastique- l'évêque de Huesca-, un militaire et le régent de l'Audience), qui désigna Palafox comme son président. Bien que l'on ait convoqué les Cortès pour célébrer une seconde séance le 14 juin, elles ne se réunirent plus et la même chose arriva avec la Junte. Vers la fin du mois de juillet, on convoqua une nouvelle Junte de caractère consultatif où figuraient les membres élus aux Cortès pour cautionner ainsi les décisions de Palafox ${ }^{17}$.

Huesca, où fut assassiné le gouverneur Clavería au début du mois de juin, forma sa propre Junte suivant l'exemple de Saragosse. Teruel en fit autant. Sa Junte avait trentesix membres, représentants du clergé, des autorités et des nobles parmi lesquels se distinguèrent les libéraux Antillón et Romero Alpuente ${ }^{18}$. On peut être étonné du fait que, pour revivifier la résistance de l'Aragon après la perte de Saragosse, la Junte Suprême Centrale ordonna le 18 juin 1809 à la Junte de Molina de Aragón (Province de Guadalajara) qu'elle s'unisse à celle d'Albarracín, Téruel, Daroca, Calatayud et Moya pour former la junte Supérieure d'Aragon laquelle, à partir de ce moment-là, assuma le titre de « Junte Supérieure d'Aragon et d'une partie de Castille ${ }^{19}$.

Les Juntes de Catalogne

Les Juntes locales et municipales (Lérida, Tortosa Vic, Mataró, Gérone, Manresa, Cervera, Solsona, Tarragona, La Seu d'Urgell, Puigcerdà, Vilafranca del Penedès et Granollers ) furent les premières à se former. Ce fut ensuite la Junte Suprême de Catalogne qui s'installa à Lérida le 18 juin et à laquelle assistèrent douze des membres nommés par les Juntes municipales. En réalité, elle se constitua dans le sillage de l'Aragon et sa légitimité se fonde sur la représentation municipale qu'elle avait. Son premier objectif fut de former une armée de 40000 hommes et d'administrer les domaines des Finances et de la Justice. Elle interdit aux Juntes de s'approprier des dîmes et des prémices de l'Église, des cens et rentes seigneuriales, dans le but de supporter les frais militaires, et réprima à tout moment toute violation de ces droits. Ses heurts avec les autorités militaires, les capitaines généraux, furent constants. Il suffit de rappeler que pas moins de huit généraux passèrent par la Catalogne pour remplir cette charge entre 1808 et 1812 : le marquis de Palacio, Juan Miguel de Vives, Reding, Blake, Enrique O'Donnell, Carlos O'Donnell, Iranzo et Lacy. La Junte Suprême fut la première à se saborder par ordre de Luis Lacy à partir du 1er décembre 1812. Les échecs militaires successifs et les problèmes économiques et financiers forcèrent la célébration des Congrès de Manresa (1809), Solsona (1810) et Tarragona (1811) qui n'apportèrent pratiquement aucune solution. 
30 Des treize principales Juntes Catalanes citées avec un total de 218 membres, 68 appartiennent au clergé, (31,1\%), 31 à l'armée (14,2 \%), 22 à la noblesse (10\%), 4 aux propriétaires terriens (1,8\%), 19 aux municipalités, (8,7 \%), 8 aux commerçants $(3,6 \%)$, 4 aux notaires $(1,8 \%), 5$ aux avocats $(2,2 \%), 57$ aux métiers ou à la paysannerie $(26,1$ $\%)^{20}$.

\section{La Junte de Majorque}

31 Face aux nouvelles qui arrivèrent de Majorque au sujet du soulèvement de Valence et à cause de la pression populaire, le capitaine général se vit obligé de convoquer le 29 mai une Junte extraordinaire à laquelle assistèrent des représentants des forces vives de la ville (généraux et officiers militaires, intendant, proviseur ecclésiastique, régent et ministres du tribunal, régisseurs de la ville et deux chanoines). Après une longue discussion, on décida de déclarer la guerre à la France et de former une Junte Suprême de gouvernement composée de toutes les autorités du Royaume de Majorque où résiderait toute l'autorité.

Après le Te Deum officiel à la cathédrale, le 30 mai, se réunirent les vingt- cinq personnes désignées pour faire partie de la nouvelle institution créée (cinq représentants de l'armée, quatre de l'Église, six de l'Audience, six de la municipalité, deux commerçants et deux nobles). Elle forma bientôt deux commissions pour traiter les affaires militaires et des Finances et sollicita l'aide anglaise. Ses relations avec les militaires furent compliquées surtout au moment où $\mathrm{F}$. de la Cuesta fut désigné capitaine général en décembre 1808 et refusa d'assister aux séances de la Junte. L'arrivée du comte de Amayans, nommé commissaire par la Junte de Majorque compta avec la représentation des autres îles (Minorque et Ibiza) ${ }^{21}$.

Les Juntes de Valence

33 Après l'incident du 23 mai, le célèbre épisode du Palleter, le 24 se produisit la prise de la Ciudadela, ce qui permit au peuple de s'armer ${ }^{22}$. Le lendemain, se constitua la Junte et on réunit sa première séance l'après-midi même. Plus de cinquante personnes firent partie de la Junte, au total : le capitaine général, comte de la Conquista (président) ; cinq membres de l'Audience; treize ecclésiastiques; cinq représentants de la ville; quatre nobles, quatre pour l'ordre des avocats, quatre militaires, deux représentants des commerçants en gros; deux pour le petit commerce; deux pour les artisans et quatre paysans de la Huerta. Outre ces membres, on considéra comme membres sept militaires, le comptable de l'armée (Canga Argüelles) et le gendarme principal ${ }^{23}$.

Les difficultés croissantes de toute sorte et le besoin de chercher un remède aux problèmes du moment (1810) poussèrent le général Bassecourt à convoquer un Congrès Provincial du Royaume, imitant ainsi ceux qui avaient existé en Catalogne. Au total, la Junte-Congrès serait formée par vingt-deux individus outre le capitaine général et les membres de la Junte précédente. Vers le début de juillet 1811, le général O’Donnell supprima la Junte-Congrès et en établit une nouvelle : la Junte Supérieure de Province et Gouvernement. Le 22 juillet, le nouveau capitaine général marquis del Palacio nomma à son tour une Junte Régimentaire ${ }^{24}$.

Le 28 mai 1808, on publia à Castellón le ban ordonnant l'enrôlement général expédié par le Royal Accord. Tout de suite après, on constitua une Junte particulière dépendant de Valence, sous la présidence du marquis de Usátegui. À Alicante, une fois connu le soulèvement de Valence, on constitua le 28 mai une Junte de Gouvernement. Les membres qui font partie de la Junte sont les mêmes que ceux de la municipalité (gouverneur militaire et corrégidor, régisseurs et députés du peuple) et d'autres 
personnes appartenant à la noblesse, au clergé et au commerce parmi lesquelles on remarque M. Antonio Valcarcel (Prince Pio et marquis de Castel Rodrigo). On nomma, à la demande du peuple, deux nouveaux membres représentants de la Junte, les prêtres José Maluenda et Angel Verdaguer. On exigea la sortie de la Junte de I. Spering, suspecté d'être un "afrancesado». Il fut toutefois réadmis plus tard par ordre de la Junte Supérieure de Valence après avoir démontré son adhésion à la cause patriotique. La Junte d'Alicante fut dissoute le 20 octobre 1808. À partir de cette date, c'est le Conseil municipal ordinaire qui se réunit. La municipalité avait clairement conscience d'avoir assumé à nouveau toutes ses attributions ou facultés. Les relations de la municipalité d'Alicante avec la Junte Suprême du Royaume ou Commission du Royaume, constituée au début de l'année 1812 et installée à Alicante après la chute de Valence sous la domination française, ne furent pas de toute cordialité. C'est alors que les problèmes surgirent, à cause du paiement des contributions, et on nomma pour cela un commissaire aux comptes en vue de résoudre le litige entre les deux organismes.

Les Juntes de Navarre et de la Rioja

37 Le 1er juin, les habitants d'Estella se prononcèrent en faveur de Ferdinand VII, incitant tous les villages du bailliage à se joindre à eux. Tafalla, Puente la Reina, Viana, Villafranca, Cáseda, Lodosa et Mañeru suivirent l'exemple. D'autres localités (Azagra, Arroniz, Mendivia, Dicastillo, etc.) refusèrent cependant les invitations au soulèvement suivant les recommandations de la "Diputación». Cet organisme traditionnel proposa au vice-roi de créer une Junte semblable aux Juntes provinciales, composée par deux membres du Conseil, deux de la Royale Cour, deux régisseurs de la ville ou prébendiers de la cathédrale, deux députés et d'autres personnes désignées par le vice-roi. Cependant le Conseil, en tant que Haut tribunal de Navarre, émit un rapport négatif à ce sujet car, à son avis, cette Junte portait préjudice à la Constitution du Royaume. C'est pourquoi elle ne se constitua jamais et que la «Diputación» continua d'exercer son pouvoir. Le soulèvement n'eut de succès qu'à Tudela et le 2 juin on célébra une séance extraordinaire à laquelle participèrent le maire, les régisseurs, l'évêque, certains chanoines et plusieurs habitants qui se constituèrent en Junte ${ }^{25}$.

Logroño se trouva bientôt sous la domination française. Bien que la ville se souleva en armes le 30 mai et que le 2 juin on ordonna un enrôlement général, les insurgés, principalement des paysans des villages des environs ne purent contenir la poussée des troupes de Verdier. Grâce à l'action de l'évêque de Calahorra, la ville ne fut pas livrée à la mise à sac habituelle, mais il fallut pour cela payer une contribution de 120000 réaux. Le gouvernement de la ville fut en 1809 aux mains de sa municipalité formée par douze individus, cinq pour la noblesse, six pour le tiers état, deux députés du commun, plus le syndic général. Pendant l'occupation napoléonienne, on renouvela la municipalité en attendant l'instauration de la municipalité constitutionnelle en 1814.

Dans les territoires contrôlés par les patriotes, on constitua plusieurs Juntes comme celle de Soto en Camareros en septembre 1809, composée de quatre membres. On forma, suivant les directives de la Junte de Molina de Aragón, celles d'Arnedo, Enciso, Cornago et Covaleda dans la province de Soria. Certaines bandes de guérillas, très nombreuses à partir de 1809 , dépendaient de ces Juntes. Elles occasionnèrent de nombreuses querelles portant sur les limites des juridictions des Juntes, notamment avec le commissaire royal des deux Castilles, le marquis de Barrio Lucia. Les incidents incessants et les excès des guérilleros incitèrent les députés de Logroño à solliciter à Séville le 4 décembre 1809 la création d'une Junte pour la Rioja qui fut concédée le 14 
du même mois. Cette nouvelle Junte se forma à partir d'un représentant de la Junte de Soto en Camareros, plus un membre de la province d'Alava et des Juntes d'Arnedo, Enciso et Covaleda, sous la présidence du capitaine de vaisseau M. Ignacio Marrón qui avait été membre de la Junte d'armement de Nájera. Elle fut dissoute par Canga Argüelles le 6 décembre $1811^{26}$.

Les Juntes de Castille et de la Manche

40 À Tolède on constitua le 18 juin 1808, après l'abandon de la ville par les Français, une Junte appelée «Permanente de Tranquillité Publique» formée par 36 membres: l'archevêque Luis de Borbón (président), son secrétaire, treize ecclésiastiques, l'intendant, le régent, des représentants de la municipalité, militaires, avocats, commissaire de guerre, comptable des rentes royales et certains artisans et commerçants. Son but principal était, bien évidemment, de contrôler l'action de la "populace" et bien moins d'organiser la résistance. Elle fut même obligée d'ordonner plusieurs incarcérations face à la série d'actes de vandalisme qui se produisirent dans les derniers jours de juillet. Pendant toute cette période, elle eut une attitude profrançaise et elle ne se convertit au patriotisme, comme le cardinal Bourbon, qu'après l'abandon de Madrid de Joseph 1er le 29 juillet et l'arrivée des héros de Bailén en l'honneur desquels on célébra des festivités extraordinaires ${ }^{27}$.

41 L'influence dans la Manche de la mutinerie d'Aranjuez fut pratiquement instantanée, comme le prouve la détention de Pepita Tudor (l'amie intime de Godoy) à Almagro. Il est vrai qu'elle fut libérée ensuite par l'intermédiaire des autorités françaises. À partir de ce moment-là, dans la majorité des villes de la Manche, se formèrent des Juntes locales (gouvernementales ou de défense). Elle n'étaient certes pas très puissantes, comme on peut le déduire à partir des tentatives de la Junte de Grenade pour les dominer ou les inclure dans leur zone d'influence. Celle-ci envoya des mandataires à Ciudad Real, Alcaraz, Daimiel, Almagro, Almodóvar, Villanueva de los Infantes et Alcazar de San Juan.

Dans un grand nombre de cas, comme à Manzanares et Valdepeñas, les Juntes sont les mêmes Conseils municipaux qui assument leurs compétences, influencés et contrôlés par les individus étrangers à ceux-ci, et cela à cause de la pression populaire. À Campo de Criptana, la Junte se vit obligée d'être accommodante avec les insurgés en attendant d'être en mesure d'imposer son autorité. À Villacañas, il semblerait que ce fut le secteur le plus radical qui s'imposa. Dans le cas de Cuenca, ce fut l'évêque qui maintint l'esprit de l'insurrection, car le corrégidor et l'intendant avaient été incarcérés et leurs maisons mises à sac. La Junte de défense de la Manche s'institua à Ciudad Real et fut présidée par l'intendant Juan Módenes de la Torre. Le soulèvement de Valdepeñas se produisit le 6 juin. Le peuple fit face aux envahisseurs français commandés par le général Liger-Belair jusqu'à ce que la Junte de défense, formée par d'anciennes autorités avec d'autres nouvelles (le curé "Calao") et du peuple (le contrebandier Juan Madero), accorde de cesser la lutte du fait du grand nombre de pertes ${ }^{28}$.

Guadalajara et Molina de Aragón formèrent leurs Juntes respectives. Celle de la capitale fut bientôt obligée de s'établir à Sigüenza à cause de l'occupation ennemie qui présida son évêque. Les affrontements entre la Junte de cette ville et celle de Guadalajara pour des questions d'organisation de la défense furent continuels. Pour sa part, le guérillero et brigadier Juan Martinez Díez (El Empecinado, l'obstiné) affronta la Junte de Guadalajara, demanda à la régence en 1811 la formation d'une Junte Supérieure de 
Nouvelle-Castille ou Royaume de Tolède "pour éviter ainsi les jalousies et les discordes de certaines Juntes avec d'autres ", mais il n'eut aucun succès ${ }^{29}$.

Les Juntes de Murcie

44 Carthagène fut le premier foyer du Royaume de Murcie où la révolution éclata. Dès l'après-midi du 23 mai et jusqu'au lendemain midi, le peuple mutiné se rendit maître des rues et obligea la municipalité réunie à proclamer officiellement le roi espagnol. Le peuple demanda des armes et obligea les chefs des régiments à jurer fidélité à la patrie. Comme garantie de l'exercice du pouvoir, les émeutiers nommèrent deux commis de la Junte Générale (B. Hidalgo et G. Ciscar) pour résoudre les questions pertinentes. Ils destituèrent de sa charge le capitaine général Borja et le gouverneur de la place (Butler) et, le 24, on constitua une Junte particulière ou gouvernementale présidée par le marquis de Camarena la Real (nouveau gouverneur) et formée par le capitaine général (B. Hidalgo), les principales autorités de la marine (parmi celles-ci, se trouvait Ciscar) les chefs des régiments, les représentants de la municipalité et les représentants directs du peuple. Par ailleurs, le 28 on constitua une Junte de Guerre présidée aussi par Camarena et d'autres autorités militaires. Le 10 juin, un nouveau tumulte coûta la vie au général Borja, accusé d'être un " afrancesado " .

Après le soulèvement de Cartagena se produisit celui de Murcie le 24 mai. La pression populaire obligea la municipalité à se réunir et une Junte fut ainsi constituée. Elle était présidée par Floridablanca et quatorze membres, représentants de la noblesse, milice, ecclésiastiques (parmi eux l'évêque et le doyen) et les autorités municipales. Albacete suivit les recommandations de Murcie et la municipalité se constitua elle même en Junte.

46 Les villages de Murcie créèrent leurs Juntes respectives. Les juntes de Calasparra, Moratalla et Caravaca exigèrent que les conscrits n'abandonnent pas les villages, organisant la défense du territoire avec la totalité de leurs habitants sans distinction de classes sociales $^{31}$.

Les Juntes de Castille et León

47 Salamanca forma une Junte le 4 mai, présidée par le marquis de Cerralbo et regroupant onze membres, le comte de Vallagonzalo, le maire principal, le compteur de la province, l'évêque et un représentant de la municipalité, un du Commun, un de l'Université, un du Chapitre et un de la Chapelle Royale de San Marcos en plus du secrétaire. Quelques jours plus tard, on y incorpora de nouveaux membres : le vicomte de Revilla et R. Benavente comme régisseurs, deux militaires et deux chanoines. La Junte de Salamanque, de composition variable, prolonge en quelque sorte la représentation traditionnelle de la société de l'Ancien Régime ${ }^{32}$. À Ciudad Rodrigo, on forma une nombreuse Junte le 5 juin composée de trente-cinq membres, la moitié d'entre eux ecclésiastiques. Quelques jours après, se produisit un tumulte populaire que ni la Junte ni l'évêque ne furent capables d'apaiser et qui provoqua la mort du gouverneur L. Marínez de Ariza et de plusieurs sympathisants des Français ${ }^{33}$.

La Junte Provinciale de Soria se constitua le 3 juin 1808, fruit de l'action spontanée des masses populaires sous la présidence du commandant des armées royales F. de Paula Carrillo $^{34}$. Parmi les vingt-et-un membres qui la formaient, la plupart étaient des défenseurs de l'Ancien Régime. Il existait une représentation des charges institutionnelles: corregidor, intendant général de province, régisseurs de la ville, proviseur général, député de ravitaillement, procureur de l'État du commun et proviseur de l'université de la Terre. Remarquons parmi eux des représentants de 
l'Église. Et en dernier lieu se trouvent les représentants des nobles et de l'armée, le brigadier des armées royales, chevaliers militaires et chevaliers de la noblesse. Le 9 juin, la Junte Provinciale de Soria fut dissoute et l'on forma une Junte d'Armement et de Défense, suivant les instructions du capitaine général G. de la Cuesta.

À Valladolid, ce fut la pression populaire qui obligea le capitaine général Gregorio de la Cuesta à ordonner un recrutement obligatoire et à convoquer une Junte d'Armement et Défense le 2 juin 1808, sous sa présidence, à laquelle furent appelés deux représentants de chaque corporation. Quelques jours plus tard, il élargit cet ordre aux provinces restantes $^{35}$. Palencia suivit le modèle de Valladolid imposé par le capitaine général. Après la réunion extraordinaire, célébrée à la mairie, à laquelle assistèrent les autorités et les personnalités civiles et ecclésiastiques le 1er juin, une Junte d'armement fut créée pour la défense de la province, présidée par l'ancien général Diego de Tordesillas ${ }^{36}$.

À Zamora, le peuple désigna le 31 mai quatre représentants rattachés aux corps militaires qui se présentèrent chez le corregidor et exigèrent de lui de brûler l'ordre du duc Berg et d'armer le peuple. Immédiatement, on constitua une Junte d'Armement et de Défense présidée par l'évêque et formée par quinze membres : cinq représentants du clergé, quatre de l'armée, trois de l'ancienne administration et trois représentants du tiers état ${ }^{37}$.

51 À Segovia, se forma tout d'abord une Junte dénommée Civile et Militaire le 3 juin, composée par les autorités civiles et militaires et par les représentants de la municipalité. Le 8, on forma une Junte d'Armement et de Défense de la province, laquelle comprenait une importante représentation ecclésiastique ${ }^{38}$. Burgos forma de la même manière une Junte d'Armement et de Défense de sept individus, qui organisa le recrutement général de la province au moyen d'une exhortation ${ }^{39}$. Il faut remarquer qu'en mars 1812 quatre de ses membres furent incarcérés par l'armée française et, après avoir été conduits à Soria, ils furent exécutés et leurs cadavres furent pendus pour servir de leçon à ces villages.

52 À León, le peuple s'érigea en protagoniste du soulèvement du 27 mai, obligeant les autorités civiles et ecclésiastiques à décider la formation le 30 d'une Junte composée de seize individus, des autorités provinciales et des membres de la municipalité sous la présidence du commandant de la province $M$. Castañon. Après avoir ordonné le recrutement, par la pression populaire, il accéda à ce que les citoyens aient six représentants ${ }^{40}$.

53 Le 22 juin, se constitua la Junte Provinciale présidée par Antonio Valdés et huit représentants de la municipalité, en plus des sept nommés par la grande Junte (de l'administration provinciale), six par le peuple (un commerçant, un avocat, un propriétaire terrien, le secrétaire de la mairie et deux administrateurs de rentes), ainsi que les membres désignés par la Junte Générale (un chanoine et deux chevaliers), sept représentants des partis et autant de députés des provinces de la Vieille Castille ${ }^{41}$.

54 Après la défaite de Rioseco, les Juntes de León et de la Vieille Castille, qui avaient fusionné depuis le 21 juillet, se rendirent à Ponferrada où elles s'accordèrent pour envoyer Tadeo Manuel Delgado afin d'obtenir un traité d'union avec les Juntes de Galice et des Asturies qui fut signé le 10 août 1808, mais sans la Junte des Asturies. Pendant ce temps-là, à León, s'est formée une autre Junte parallèle sous les auspices du marquis de Portazgo (général F. de Paula Gómez de Teron). Les relations de la Junte provinciale avec le capitaine général Gregorio García de la Cuesta furent très mauvaises. Les procédés arbitraires du général arrivèrent à un point tel qu'il incarcéra Antonio Valdés 
lui-même et le vicomte de Quintillana alors qu'ils se dirigeaient à Ocaña pour former la Junte centrale en représentation du León et de la Castille. Astorga constitua sa Junte d'Armement et de Défense sous la présidence de l'évêque, formée par douze individus représentants de l'état nobiliaire, douze autres de l'ordre ecclésiastique et quatre du tiers état ${ }^{42}$.

Les Juntes de Galice

55 À la Corogne, la population se méfiait de ses autorités. Le 30 mai, après l'arrivée du représentant des Asturies et de l'étudiant du León qui informèrent des soulèvements des deux provinces, se produisit l'assaut contre l'arsenal et se constitua le soir même une Junte d'Armement et de Défense rattachée à des personnes de l'administration précédente : onze militaires, onze représentants de l'Audience, neuf ecclésiastiques, six de la municipalité, trois commerçants et les autres personnes.

À Santiago de Compostelle, s'est formée le jour même, le 30 mai, à la demande de l'archevêque, une Junte d'Armement et de Défense, composée par dix-neuf membres: six membres du clergé, un représentant de la municipalité, trois commerçants, trois de l'Université, trois gentilshommes (rentiers) et trois autres personnes (militaires et juges). Les élus furent proposés par l'officier Armisen comme la dénonciation présentée par la municipalité au gouverneur militaire de La Corogne ${ }^{43}$. À Vigo, on forma aussi une Junte, dite subalterne et consultative, où prédominaient clairement les représentants de l'armée et de la fonction publique (six militaires, deux nobles, deux commerçants, un ecclésiastique, le capitaine du port, l'administrateur de rentes et le sergent major de la place). Les tensions entre la Junte de La Corogne et celle de Santiago pour le contrôle économique et militaire furent continues ${ }^{44}$.

Le 5 juin, fut instaurée à la Corogne la Junte Suprême de la Galice avec un représentant de chacune des sept villes les plus importantes. Il participa, entre autres, à l'organisation militaire et financière, à l'appui au soulèvement portugais, à la pétition d'aide à l'Angleterre et au rapprochement avec les autres Juntes. Précisément avec celle de León et Castille, il signa un traité d'union le 10 août 1808, de courte durée car il ne fut en vigueur que jusqu'en septembre de cette année. Lorsque la Junte de Galice disparut en janvier 1809, à cause du contrôle du territoire par les Français, on constitua la Junte de Lobeira dans le district de Bande, appartenant à Orense, sous la présidence de l'évêque d'Orense Pedro Quevedo et cinq autres personnes (militaires, licenciés et un greffier). À partir du mois de mai, le maréchal marquis de la Romana, de tendance absolutiste, prit le contrôle politique de Galice. Mais on créa une Junte de subsides, à l'abri du Règlement sur les Juntes du 17 décembre 1809.

Autres Juntes

58 À Santa Cruz de Ténérife, on constitua une Junte dans les premiers jours de juillet 1808, à la demande de la Junte Sévillane. On forma une Junte Supérieure de Canaries qui fut en vigueur entre 1808 et juillet 1809. M. Alonso de Nava Grimón, marquis de Villanueva del Prado en fit partie et fut désigné, avec l'opposition, certes, de Gran Canaria, pour représenter les Îles Canaries à la Junte Centrale. On peut, grâce aux écrits et aux Mémoires de ce personnage, entrevoir le combat livré par La Laguna et Las Palmas pour devenir la capitale des Îles Canaries qui finalement échut à Santa Cruz de Ténérife ${ }^{45}$.

Santander constitua sa Junte le 27 mai. Elle était composée par des individus de la municipalité et des personnalités influentes de la ville, sous la présidence de son évêque Rafael Menendez de Luarca, Régent de Cantabria qui jouissait d'une grande popularité. La Junte provinciale eut la représentation des députés de chaque 
juridiction. À Bilbao, le 4 août, se constitua, par influence de Santander, une Junte gouvernementale composée par deux régisseurs, quatre avocats, deux prêtres, le maréchal de camp Zarauz, deux députés généraux et le maire. Plus tard, le soulèvement de Bilbao s'étendit aux différents villages de la Seigneurie de Biscaye. Vers la mi-août, les Français pénétrèrent dans la capitale et la saccagèrent, comme ils le firent à Abando, Begoña et Deusto ${ }^{46}$.

Lorsque la Junte Centrale de la province de Madrid se retira, il se forma une Junte de Défense en décembre 1808, présidée par le duc del Infantado, avec comme membres le lieutenant général Tomas Morla, le maire de la ville, des corrégidors, l'intendant et le marquis de Castellar. Face à cette situation si critique, la Junte se réunit en séance permanente dans la Casa de Correos (Maison des Postes) et distribua à la population les armes gardées dans l'Armurerie Royale. Femmes et enfants travaillèrent avec ardeur au dépavage des rues, à creuser la terre pour s'en servir afin de remblayer les portes et les portillons ${ }^{47}$. Les Mémoires de l'époque se réfèrent au fait que, lorsque le peuple se rendit compte que les cartouches fournies par le marquis de Perales étaient vides, celui-ci fut tué et les femmes, les enfants et les moines remplirent à nouveau les cartouches.

Dans les colonies américaines, comme dans la péninsule, on créa des Juntes pendant la Guerre d'Indépendance qui remplacèrent les conseils municipaux coloniaux, les audiences, les vices-rois ou les capitaines généraux, et qui se constituèrent comme de nouveaux organes de pouvoir. Au Portugal, la dissolution de la Régence en février 1808 et les événements d'Espagne servirent de modèle pour la formation de Juntes. Tout au long des mois de juillet et d'août, les Juntes s'étendent dans le pays tout entier, de la périphérie au centre, prenant comme modèle celle créée à Porto le 18 juin, présidée par l'évêque et celles créées dans les provinces espagnoles limitrophes (Salamanca, Ciudad Rodrigo, Badajoz, Séville, Cadix et Ayamonte) avec lesquelles elles établirent d'étroites relations ${ }^{48}$.

Peu importe que les Juntes que nous avons analysées contiennent en leur sein de nombreuses contradictions, surtout si nous nous limitons à la composition de leurs membres ou à leurs décisions qui défendent toujours l'ordre social en place. Malgré cela, les Juntes jouèrent un rôle d'importance vitale dans la révolution politique espagnole qui commence en 1808. Se dotant de nouveaux pouvoirs, les Juntes provinciales d'abord, après la Junte Centrale et, ensuite, le Conseil de Régence, le processus politique ouvert débouche finalement dans les Cortès extraordinaires réunies à Cadix d'où est issue la Constitution de 1812. Le processus de désignation de ses membres dans de nombreux cas par acclamation a un précédent plus immédiat dans les élections des procureurs ou des députés du Commun à partir de 1766, bien que dans très peu de Juntes nous trouvions une représentation authentique du peuple que l'on fit bientôt taire, comme à Alicante ou à Majorque. Il ne faut pas oublier que, lorsqu'on envisagea la formation d'une Junte Centrale, de nombreuses Juntes manifestèrent la nécessité d'une réforme politique, quoique extrêmement timide, pour faire face comme le déclara la Junte de Séville - à la concurrence du programme réformiste et "afrancesado" de l'Assemblée de Bayona. Les Juntes servirent de toute manière pour diffuser la politique et devinrent ainsi des intermédiaires culturels capables de politiser la population. Il faut ainsi voir les Juntes comme des instruments de modernisation politique et de création de l'opinion publique. 
63 En dépit de leur ambiguïté, les Juntes furent sans aucun doute le moteur du changement politique à partir de la base et la plate-forme d'action entre les différentes classes sociales. Dans l'imaginaire collectif créé par le libéralisme, le mouvement des Juntes de 1808 symbolise la révolution espagnole et il est devenu l'un des instruments de base du changement politique et social de l'Espagne du XIXe siècle. Le schéma de la révolution des Juntes se répètera dans toutes les conjonctures révolutionnaires et crises politiques qui se succédèrent tout au long du processus de la Révolution libérale entre 1808 et 1843 et aussi pendant la période d'établissement et de crise du nouvel État jusqu'à la Révolution de 1868.

\section{NOTES}

1.Cette étude fait partie du projet BHA 2001-2509 financé par la Dirección General de Investigación Científica y Técnica.

2.A. Moliner Prada, « La conflictividad social en la Guerra de la Independencia », Trienio, no.35, mai 2000.

3.F. J. Maestrojuan Catalán, « entre la sobrerrevolución y la contrarrevolución: la cultura política de los prohombres zaragozanos en el tránsito a la modernidad, ", Cuadernos de Investigación Histórica, no. 18 (2001), p. 37; F. X. Guerra, Modernidad e independencia. Ensayos sobre los revoluciones hispánicas, Madrid, 1992, pp. 160-161.

4.F. Carantoña Alvarez, « Poder e ideología en la Guerra de la Independencia », Ayer, no 45 (2002), p. 284.

5.Id., p. 279.

6.R. Hocquelet, Résistance et révolution durant l'occupation napoléonienne en Espagne 1808-1812, Paris, 2001, pp. 148-149. Cet auteur distingue le processus de formation des Juntes du soulèvement et de la mobilisation populaire.

7.A. Von Schepeler, Histoire de la révolution d'Espagne et de Portugal ainsi que de la guerre qui en résulta, Liège, 1829, vol.I, p. 77.

8.J. De Haro Malpesa, Guerra de la Independencia. La Mancha 1808, Ciudad Real, 2000, p. 54. 9.J.-R. Aymes, « Las nuevas autoridades: las Juntas. Orientaciones historiográficas y datos recientes ", dans Actas del Congreso Internacional El Dos de Mayo y sus Precedentes, Madrid, 1992, pp. 567-581.

10.R. Alvarez Valdés, Memorias del levantamiento de Asturias en 1808, Oviedo, 1898 ; F. Carantoña Alvarez, La Guerra de la Independencia en Asturias, Gijón, 1984 et Revolución liberal y crisis de las instituciones tradicionales asturianas, Gijón, 1989.

11.La meilleure étude au sujet des événements de mai est celle de F. Aguilar Piñal , « El mes de mayo de 1808 en Sevilla ", dans J. F. Fuentes et Ll. Roura (éd.) Sociabilidad y liberalismo en la España del siglo XIX.. Homenaje a Alberto Gil Novales, Lleida, 2001, pp.13-35. M. Moreno Alonso, La revolución "santa" de Sevilla: la revuelta popular de 1808, Sevilla, 1997, les analyse également à partir d'un point de vue différent.

12.D. González Cruz, De la revolución francesa a la Guerra de la Independencia. Huelva a finales de la Edad Moderna, Huelva, 2002, pp. 29-40.

13.M. López ; I. Lara, Entre la guerra y la paz. Jaén (1808-1814), Granada, 1993, pp. 76-84. 
14.A. Gallego Burín, Granada en la Guerra de la Independencia, (Edic. Facsímil), Granada, 1990 , p. 17.

15.M. Reder Gadow, « Ambiguëdad de la Iglesia malagueña durante la Guerra de la Independencia », dans J.A. Armillas (dir.) La Guerra de la Independencia. Estudios, II Zaragoza, 2001, pp. 677-681.

16.J. García Pérez Y F. Sánchez Marroyo, « Guerra, reacción y revolución (1808-1833) », dans Historia de Extremadura, tome IV, Los tiempos actuales, Badajoz, 1985, pp. 652-659. 17.H. Lafoz, « La contienda en Aragón. Revisión historiográfica », dans J.A. Armillas (coord.) La Guerra de la Independencia. Estudios, vol. 1, Zaragoza, 2001, pp. 73-104; J.A. Armillas Vicente, « Las Cortes de 1808. Persistencia del Reino de Aragón », dans V Premio Los sitios de Zaragoza, Zaragoza, 1991, pp. 22-26.

18.D. Gascón Gimbao, La provincia de Teruel en la Guerra de la Independencia, 1908. 19.Archivo Histórico Nacional, Sección Estado, Legajo 3.082.

20.A. Moliner Prada, « Les Juntes a la Guerra del Francès ", L'Avenç no 225 (mai 1998), p. 31.

21.Id., La Guerra del francès a Mallorca (1808-1814), Palma, 2000.

22.V. Boix y Ricarte, Historia del País Valenciano, Barcelona, 1980, vol. II, pp. 260 et sq. 23.A. Moliner Prada, « Les Juntes del País Valencià en la Guerra del Francès », dans El primer liberalisme: l'aportació valenciana, Biblioteca Valenciana, València, 2001, pp 48-61. 24.V. Genovés Amorós, València contra Napoleó, València, 1967, p. 196.

25.F. Miranda Rubio, « La Guerra de la Independencia en Navarra », dans J. A. Armillas (dir.) La Guerra de la Independencia. Estudios, vol. I, op. cit., pp. 231-233.

26.Ma Del C. Sobrón Elguea, Logroño en la Guerra de la Independencia, Logroño, 1986, pp. $163,174-175$ et 178 .

27.C. M. Rodríguez López-Brea, Don Luis de Borbón, el Cardenal de los liberales (1777-1823), Toledo, 2002, pp.151-160.

28.J. Haro Malpesa, Guerra de la Independencia., op. cit., pp. 103-104.

29.A. Cassinello Pérez, Juan Martín, “El Empecinado”, o el amor a la libertad, Madrid, 1995, pp. 119-120.

30.E. La Parra, El Regente Gabriel Císcar, Madrid, 1995, pp. 137-141.

31.G. Sánchez Romero, Revolución y reacción en el noroeste de la región de Murcia (1808-1833), Murcia, 2001, p. 188.

32.J. Zaonero, Libro de noticias de Salamanca (Ed. de R. Robledo), Salamanca, 1998, p. 44; R. Robledo « La crisis del Antiguo Régimen », dans Historia de Salamanca, IV Siglo Diecinueve, (R. Robledo dir.), Salamanca, 2001, p. 59.

33.E. Becerra et F. Redondo, Ciudad Rodrigo en la Guerra de la Independencia, Salamanca, 1988, pp. 24-26.

34.J'ai suivi l'étude de Ma C. García Segura, Soria, veinticinco años críticos de su historia, 1789-1814, vol. II, Tesis doctoral, Univ. Complutense, Madrid, 1987, (reproducción facsímil), pp. 117- 178.

35. Noticia de los casos particulares ocurridos en la ciudad de Valladolid. Año de 1808 y siguientes, Valladolid, 1808.

36.A. Ollero de la torre, "La Guerra de la Independencia y la crisis del antiguo Régimen en Palencia ", dans J. González (dir.) Historia de Palencia, tome II, Edad Moderna y Contemporánea, Diputación de Palencia, Palencia, 1995, p.159.

37.R. Prieto, «Zamora en la transición del Antiguo Régimen », dans J.C. Alba López (dir.) Historia de Zamora, tome III, La edad Contemporánea, Diputación de Zamora, Zamora, 1995, pp. 71-73. 
38.M. Barrio Gozalo, « El canónigo de la catedral D. Vicente Román Gómez », dans Tres figuras del clero afrancesado, Aix-en-Provence, 1987, p. 108.

39. Exhortación que la Junta de Armamento de la Ciudad y Provincia de Burgos dirige á sus leales habitantes para que con sus personas y bienes concurran á la defensa de la religión, de la Patria $y$ del Rey.

40.A. Moliner Prada, « Guerra de la Independencia y revuelta social: la Junta Superior de León », dans El pasado histórico de Castilla-León, vol. III, Burgos, 1983, pp. 379-392. 41.P. García Gutiérrez, La ciudad de León durante la Guerra de la Independencia, Junta de Castilla y León, 1991, pp. 170-171.

42.A. García Fuertes, « La Junta de Defensa y Armamento de Astorga y el ejército auxiliar británico del general sir John Moore en la campaña de 1808 », dans J.A. Armillas (dir.), La Guerra de la Independencia, Estudios, vol. II, op. cit., p. 823, note 6. 43.X. R. Barreiro Fernández, Historia contemporánea de Galicia, vol. 1, La Coruña, 1982, pp. 44-51

44.G. Brey, « La Galice dans la tourmente révolutionnaire (1780-1820) », dans La Révolution française et son « public » en Espagne entre 1808 et 1814, Paris, 1989, pp. 207-209. 45.A. Cioranescu (éd.), Alonso de Nava Grimón. Obras políticas, Tenerife, 1974.

46.J. C. Enríquez y E. Sesmero, « El pronunciamiento vizcaíno de 1808: actitudes patricias y revuelta popular ", dans El jacobinisme. Reació i revolució a Catalunya i a Espanya, 1789-1837, UAB, 1990, pp. 231-244.

47.J. Muñoz Maldonado, Historia política y militar de la Guerra de la Independencia contra Napoleón Bonaparte desde 1808 a 1814, escrita sobre los decretos auténticos del Gobierno por el Dr. D.(...), Madrid, 1833, vol. II, pp. 40-41.

48.J. Tengarrinha, Movimientos populares agrários em Portugal, vol. II (1808-1825), Publicaçôes Europa-América,1994, pp. 45-47 et 56.

\section{RÉSUMÉS}

Le but de cet article est de réviser les recherches menées ces dernières années au sujet des Juntes pour établir ainsi un état de la question. On ne peut pas parler de révolution populaire, car le peuple est absent des Juntes. Mais on ne peut pas analyser ces dernières sans le soulèvement populaire qui précéda leur formation. Il faut tout de suite signaler l'énorme difficulté qui existe lorsqu'il faut créer un modèle interprétatif exprimant les traits particuliers de différentes Juntes. On ne peut pas recourir à un schéma explicatif simpliste et linéaire. En dépit de leur ambiguïté, les Juntes furent le moteur du changement politique à partir de la base et la plate-forme d'action entre les différentes classes sociales. Dans l'imaginaire collectif créé par le libéralisme, le mouvement des Juntes de 1808 symbolise la révolution espagnole.

The Crisis of State and the New Authorities: the Juntas in the War of Independence The aim of this article is to review research conducted in recent years on the juntas in order to ascertain the state of the art. Reference to popular revolution is out of place since the people were left out of the juntas. But they cannot be analysed without looking at the popular uprising which preceded their establishment. It needs to be said at the outset how difficult it is to create an interpretative model describing the specific features of the different juntas. No simple linear 
scheme of explanation suffices. In spite of their ambivalence, the juntas served as the motor of change from the bottom up and the hub of action between the different social classes. In the collective imagination fostered by liberalism, the junta movement of 1808 symbolises the Spanish revolution.

INDEX

Mots-clés : Espagne, nation, patriotisme, Ancien Régime, Juntes, junte centrale, conseil de Régence

\section{AUTEUR}

ANTONIO MOLINER PRADA

Universitat Autònoma de Barcelona, Departament d'Història Moderna i Contemporània 